\title{
Velocities and Chemical Stratification in the Outermost Core
}

\author{
Satoru TANAKa and Hiroyuki HamaguCHI \\ Observation Center for Prediction of Earthquakes and Volcanic Eruptions, Faculty of Science, \\ Tohoku University, Aoba-ku, Sendai 980, Japan
}

(Received December 2, 1992; Revised July 29, 1993; Accepted July 29, 1993)

\begin{abstract}
Travel times of long-period SKS and S2KS phases recorded at the GDSN and WWSSN stations were analyzed to retrieve information about the radial structure of the outer core. We propose a new velocity model for the outermost core named KTH. The velocity at the top of the core is $8.016 \mathrm{~km} \mathrm{~s}^{-1}$, and the velocity in the outermost $200 \mathrm{~km}$ of the core is $0.11 \mathrm{~km} \mathrm{~s}^{-1}$ faster than that of HALES and RoBERTS (1971), that in the outer 200 400 km of the core is identical within $1 \%$ to that of HALES and ROBERTS (1971). We find that the inhomogeneity index $\eta$ is slightly larger than unity in the outermost $100 \sim 200 \mathrm{~km}$ of the core where a preferable value of $\eta$ just beneath the core-mantle boundary is 1.5 which corresponds to Brunt-Väisälä frequency $N$ of $8.6 \times 10^{-4} \mathrm{~s}^{-1}$. We suggest that the outermost $100 \sim 200$ $\mathrm{km}$ of the core is stably stratified. The thickness of the stable stratified layer in the present study is not far from those of $70 \sim 80 \mathrm{~km}$ thickness derived from theoretical considerations. By comparing possible lateral heterogeneity in the outermost core with temperature distribution from a geomagnetic study, we infer that a chemically stratified layer is preferable in the outermost core.
\end{abstract}

\section{Introduction}

The core-mantle boundary (CMB) is one of the most intriguing regions for geoscientists to comprehend the nature of the Earth, because the CMB is thought to be not only a source of thermal and chemical activities occurring in the Earth (for a review see JEANLOZ, 1990) but also a source of fluctuation of Earth rotation (SASAO and WAHR, 1981). Based on the result from many geophysical disciplines, thermal and chemical boundary layers at the base of the mantle are thought to be an origin of mantle plumes (LOPER, 1991). The existence of a stably stratified layer at the top of the core has been discussed with relation to the Earth's thermal history (GUBBINs et al., 1982) or the growth of the inner core (FEARN and LOPER, 1981). The mechanical and/or electromagnetic coupling between the core and mantle was also proposed through geomagnetic studies (e.g. Bloxham and GubBIns, 1985; JAUlT and Le MouËL, 1990). For further understanding of the $\mathrm{CMB}$, a precise investigation of the structure of the both sides of the CMB is necessary.

In seismology, the mantle-side of the $\mathrm{CMB}$ called the $\mathrm{D}^{\prime \prime}$ region has long been investigated by many researchers using various seismic phases, and this region has been revealed to be radially and laterally heterogeneous (for a review, see LAY, 1989). Recently, detailed analyses of the structure of the core-side of the CMB called the $E^{\prime}$ region were started by LAY and YounG (1990), Souriau and Poupinet (1990, 1991), Kohler and TANimoto (1992), and TANAKA and HAMAGUCHI (1993a) to refine the previous studies (e.g. HALES and RoBERTS, 1971; CHOY, 1977; KIND and MÜLLER, 1977). In the light of stable stratification in the outermost core, the velocity gradient and inhomogeneity index $\eta$ (BULLEN, 1975) have long been investigated (e.g. BuLLEN, 1969; Choy, 1977; MASTERs, 1979; LAY and YounG, 1990). However, their interpretations are controversial as to whether stably stratified layer really exists, or, if so, whether the stratification is caused by thermal or chemical inhomogeneity. 
In the present study, we determine velocity structure in the outer core using SKS and S2KS travel times from widely distributed seismographic stations. In the analysis of the travel times, we took into account mantle heterogeneities as well as the ellipticity in the Earth. Based on the newly determined velocity structure, we discuss the stable stratification in the outermost core, the inhomogeneity index $\eta$ related to be Brunt-Väisälä frequency (MASTERs, 1979) and chemical and/or thermal inhomogeneities.

\section{Data}

To avoid contamination of depth phases and to analyze simple waveforms, we collected about 300 long-period seismographs recorded by the Global Digital Seismograph Network (GDSN) and the World-Wide Standardized Seismograph Network (WWSSN) for 71 deep earthquakes $(h>$ $200 \mathrm{~km}$ ). Figure 1 shows the geographical distribution of the ray paths through the outer core. SmKS $(m=1,2,3)$ travel times were reported by TANAKA and HAMAGUCHI $(1993 \mathrm{a}, \mathrm{b})$ in detail. Here we briefly describe the data used in the present study.

The travel times and epicentral distances were reduced into the common source depth of 500 $\mathrm{km}$. We calculated the corrections for times and distances based on the PREM velocity structure for the period of $1 \mathrm{sec}$ (DZIEWONSKI and ANDERSON, 1981). The number of the SKS travel time data was 157 for the GDSN and 72 for the WWSSN. The observed travel times were corrected for the ellipticity of the interior of the Earth. The ellipticity correction was calculated from the formulation of DzIEWONSKI and GiLBERT (1976). The travel time corrections for the upper mantle and the lower mantle heterogeneities were calculated from the model SH425.2 by Su and
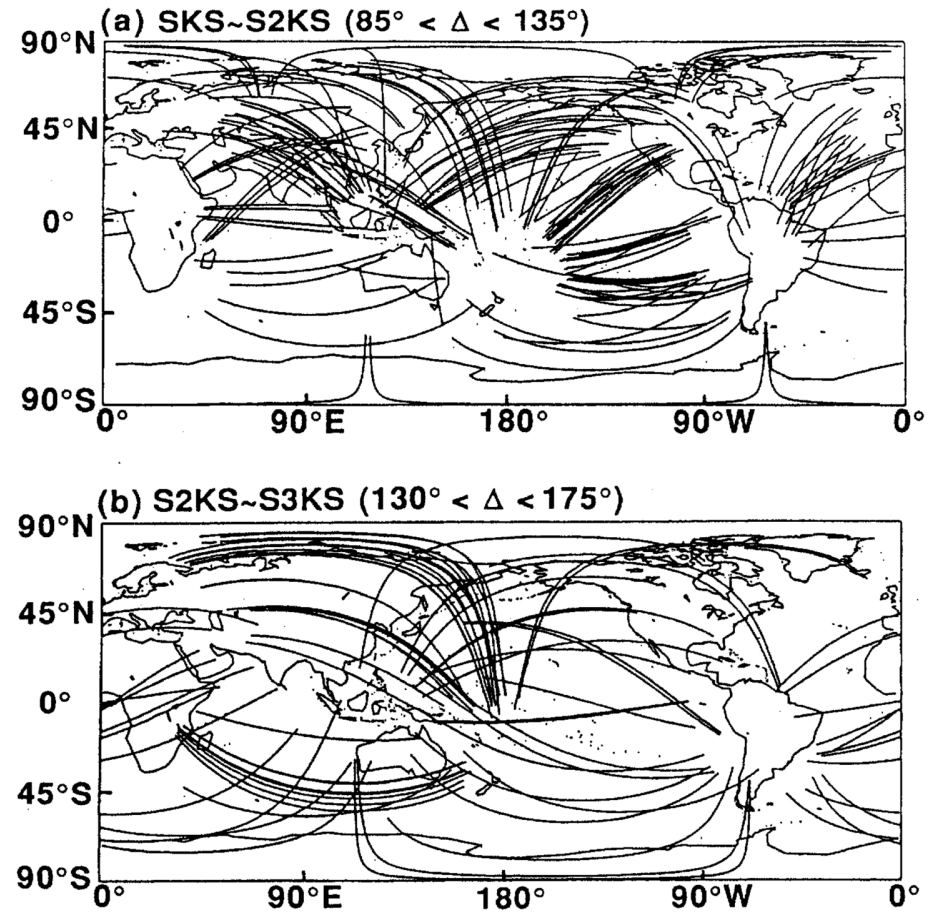

Fig. 1. Geographical distribution of great circle paths between epicenters and stations for epicentral distances (a) $85^{\circ}<\Delta<135^{\circ}$ and (b) $130^{\circ}<\Delta<175^{\circ}$. Lines represents portions of paths passing through the outer core. 
DzIEWONSKI (1991) and from the model L02.56 by DzIEwonski (1984), respectively: The P and $S$ velocity perturbations in the lower mantle were assumed to be related by $\delta v_{S}=2 \delta v_{P}$ according to REVENOUGH and JORDAN (1989). The corrected SKS travel times for the epicentral distances between $85^{\circ}$ and $135^{\circ}$ were then fitted by a quadratic polynomial. The best fitted quadratic in terms of $\Delta^{\prime}=\left(\Delta-115^{\circ}\right)$ is given by

$$
T_{S K S}=1427.52+3.771 \Delta^{\prime}-0.0404 \Delta^{\prime 2} .
$$

Figure 2 shows the corrected travel times of SKS with the curve of Eq. (1).

Previously, HALES and RoBERTS (1971) measured the differences in arrival times of S2KS and SKS. Unfortunately they did not take into account that S2KS incurred the phase shift due to the internal caustic (CHOY and RichARDS, 1975). Since all data used in their study came from the seismographic stations located in North America, their data could not provide a globally averaged structure. In the present study, we considered $\pi / 2$ phase distortion of S2KS due to the internal caustics, and measured carefully the 174 differential travel times of S2KS-SKS from the widely distributed seismographic stations of the GDSN and the WWSSN for epicentral distances of $98 \sim 135^{\circ}$. Also we obtained 71 S3KS-S2KS times for epicentral distances of $130 \sim 175^{\circ}$. Furthermore, these differential travel times were corrected for ellipticity at the core-mantle boundary (1/394) (BULLEN and HADDON, 1973), for a common source depth reduction at $500 \mathrm{~km}$ and for the lower mantle heterogeneity (DzIEwonsKI, 1984). The quadratic curves which give the optimum least squares fit to S2KS-SKS and S3KS-S2KS observations are

$$
\begin{gathered}
T_{S 2 K S-S K S}=71.74+2.933 \Delta^{\prime}+0.02438 \Delta^{\prime 2}, \\
T_{S 3 K S-S 2 K S}=44.89+1.139 \Delta^{\prime \prime}+0.00369 \Delta^{\prime \prime 2},
\end{gathered}
$$

where $\Delta^{\prime}=\left(\Delta-115^{\circ}\right)$ and $\Delta^{\prime \prime}=\left(\Delta-155^{\circ}\right)$. Figures $3(\mathrm{a})$ and $(\mathrm{b})$ show the observed differential travel times compared with Eqs. (2a) and $(2 \mathrm{~b})$, respectively.

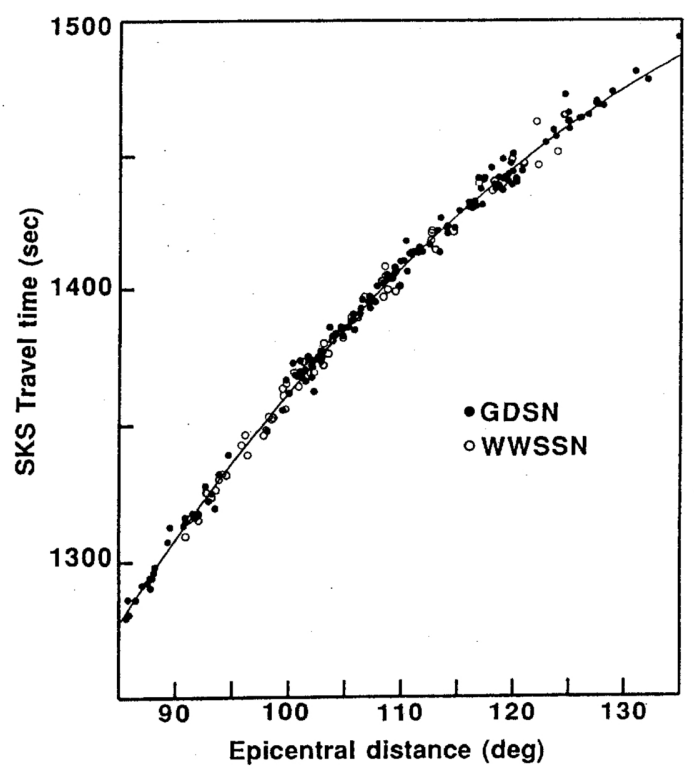

Fig. 2. Observed and the least squares fitted SKS travel times for focal depth of $500 \mathrm{~km}$. Solid and open circles indicate the observed travel times from the GDSN and WWSSN, respectively. Solid line means the least squares fitted curve of SKS travel times given in Eq. (1). 

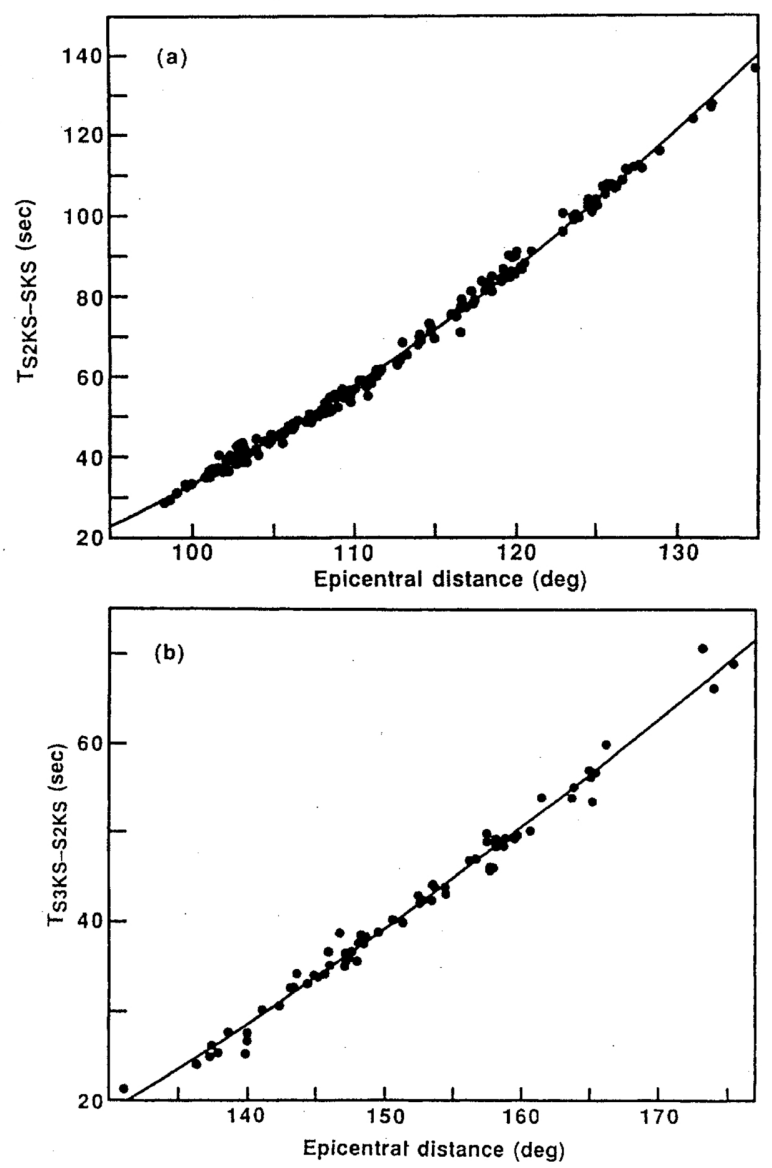

Fig. 3. Differential travel times of (a) S2KS-SKS and (b) S3KS-S2KS. Solid circle indicates the observed differential travel times. Solid line indicates the least squares fitted curve, expressed by Eqs. (2a) and (2b) for S2KS-SKS and S3KS-S2KS, respectively.

\section{Method and Results}

\subsection{Herglotz-Wiechert inversion using SKS and S2KS travel times}

Here we use a classical method for determination of seismic velocities in the outer core in a similar way did HALES and RoBERTS (1971). Slowness $p$ of SKS $\left(\mathrm{d} T_{S K S} / \mathrm{d} \Delta\right)$ is derived from a derivative of Eq. (1) with respect to $\Delta$, and that of S2KS $\left(\mathrm{d} T_{S 2 K S} / \mathrm{d} \Delta\right)$ is from a derivative of sum of Eqs. (1) and (2a). The travel times $\left(T_{k}\right)$ and distances $\left(\Delta_{k}\right)$ in the core were obtained by subtracting travel times and distances of ScS phase calculated by the PREM model from those of SKS and S2KS phases. Figure 4 shows $p$ as a function of $\Delta_{k}$ determined by this way. In order to determine $T_{k}$ and $p$ for $\Delta_{k}$ less than $20^{\circ}$, we assume the following power-law velocity distribution $v=v_{C M B}\left(r / r_{C M B}\right)^{a}$ in the outermost core. It follows that

$$
\begin{aligned}
p & =\left(\mathrm{d} T_{k} / \mathrm{d} \Delta_{k}\right) \\
& =\left(\pi r_{C M B} / 180 v_{C M B}\right) \cos \left[\Delta_{k}(1-a) / 2\right] \\
T_{k} & =\left(2 r_{C M B} /(1-a) v_{C M B}\right) \sin \left[\Delta_{k}(1-a) / 2\right]
\end{aligned}
$$




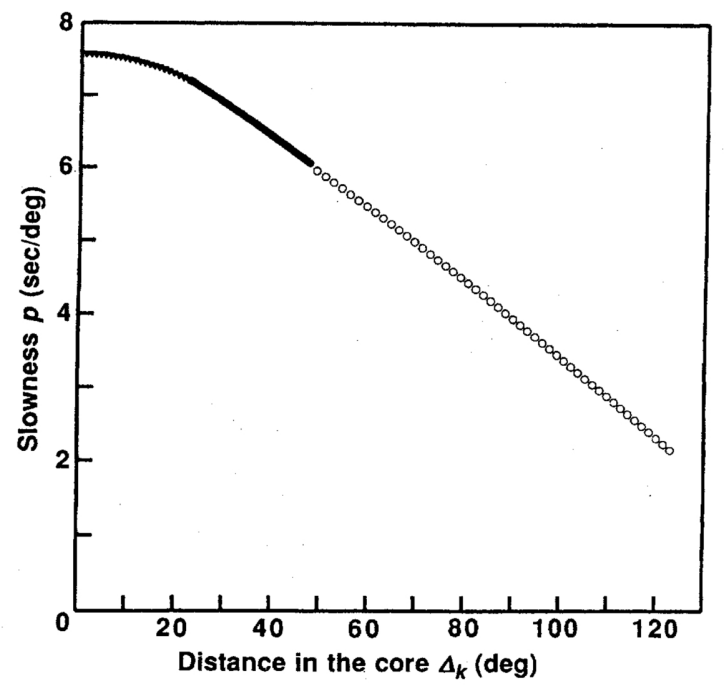

Fig. 4. Slowness $p$ in the core as a function of distance in the core $\Delta_{k}$. Open, closed circles and triangles give the values of slowness estimated from the travel time curves of SKS and S2KS, and the extrapolation assuming $v=v_{C M B}\left(r / r_{C M B}\right)^{a}$, respectively.

(RANDAll, 1970; HALES and RoberTs, 1971). Using S2KS data of $T_{k}=170.64 \mathrm{sec}, p=7.19$ sec $/ \mathrm{deg}, \Delta_{k}=22.92^{\circ}$ corresponding to the data at $\Delta_{S 2 K S}=100^{\circ}$, and $r_{C M B}=3480 \mathrm{~km}$ taken from the PREM, we find $v_{C M B}=8.016 \mathrm{~km} \mathrm{~s}^{-1}, a=1.239$ and $p$ at $\Delta_{k}=0$ is $7.577 \mathrm{sec} / \mathrm{deg}$. The extrapolated slowness $p$ is also shown in Fig. 4.

Velocity distributions in the outer core are determined from the Herglotz-Wiechert inversion based on the $p-\Delta_{k}$ curve. We obtain a model which we call KTH hereafter. The velocity distribution of the model is given in Table 1 . Figure 5 shows the $\mathrm{P}$ wave velocity of the KTH, compared with other models of the PREM, HALES and ROBERTS' (1971) and RANDALL's (1970). The P wave velocities in KTH and the Hales and Roberts' for the outermost $400 \mathrm{~km}$ of the outer core are appreciably lower than those of the PREM and the Randall's. Moreover, those in the

Table 1. $\mathrm{P}$ velocities in the outer core (Model KTH).

\begin{tabular}{cccc}
\hline $\begin{array}{c}\text { Radius } \\
(\mathrm{km})\end{array}$ & $\begin{array}{c}\text { P velocity } \\
(\mathrm{km} / \mathrm{sec})\end{array}$ & $\begin{array}{c}\text { Radius } \\
(\mathrm{km})\end{array}$ & $\begin{array}{c}\text { P velocity } \\
(\mathrm{km} / \mathrm{sec})\end{array}$ \\
\hline 1300 & 10.491 & 2500 & 9.324 \\
1400 & 10.381 & 2600 & 9.223 \\
1500 & 10.277 & 2700 & 9.119 \\
1600 & 10.177 & 2800 & 9.009 \\
1700 & 10.079 & 2900 & 8.889 \\
1800 & 9.983 & 3000 & 8.752 \\
1900 & 9.890 & 3100 & 8.609 \\
2000 & 9.796 & 3200 & 8.458 \\
2100 & 9.704 & 3300 & 8.293 \\
2200 & 9.611 & 3400 & 8.131 \\
2300 & 9.517 & 3480 & 8.016 \\
2400 & 9.422 & & \\
\hline
\end{tabular}




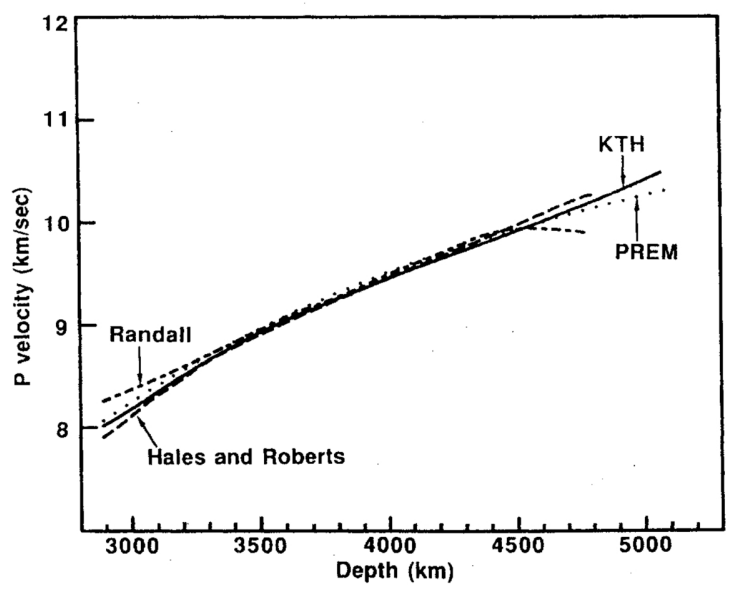

Fig. 5. P velocity distributions in the outer core of the model in the present study (the KTH model), the PREM (Dziewonski and ANDERson, 1981), Hales and RoberTs (1971) and RANDAll (1970).

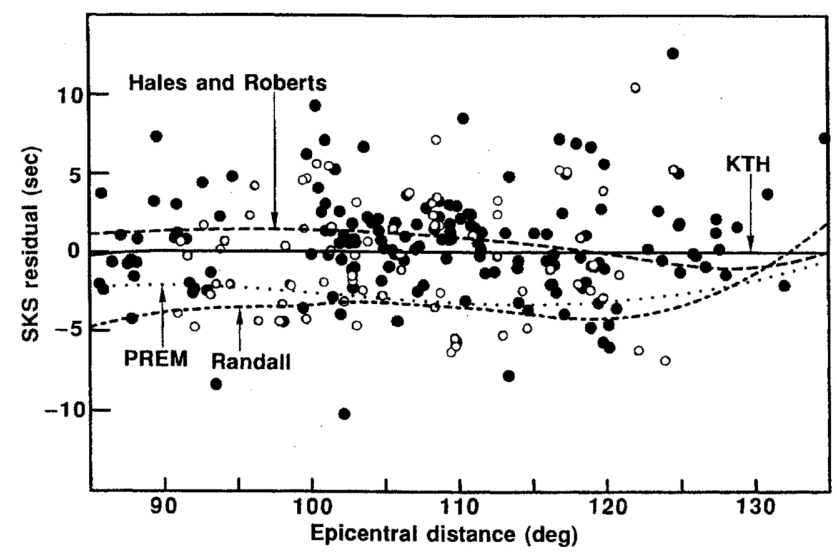

Fig. 6. Residuals of the SKS travel times as a function of epicentral distances for the models KTH, PREM, HALES and RoBERTS' (1971) and RANDALL's (1970) with respect to Eq. (1).

outermost $200 \mathrm{~km}$ in KTH are slightly faster than those in the Hales and Roberts. The KTH velocities for the depth ranges between 3500 and $4500 \mathrm{~km}$ are, at most, $2 \%$ slower than those of PREM and the Randall's.

Figure 6 shows SKS travel time residuals of the four models of KTH, HALES and ROBERTS (1971), PREM and RANDALL (1970) with respect to Eq. (1) as the reference of the observation. The travel times of SKS calculated from KTH are completely coincident with the reference, of course. The residuals of HALES and ROBERTS (1971) are within \pm 2 sec. Those of the PREM and RANDALL (1970) are 3 4 sec faster than the reference of the observation. Figure 7 shows the differential travel time residuals of the four models with respect to Eq. (2a). The $\Delta T_{S 2 K S-S K S}$ of the HALES and RoBerTs (1971) and the RANDAll (1970) are located near the upper and lower bounds of scatter of the observed residuals, respectively. Those of the PREM start to increase at 


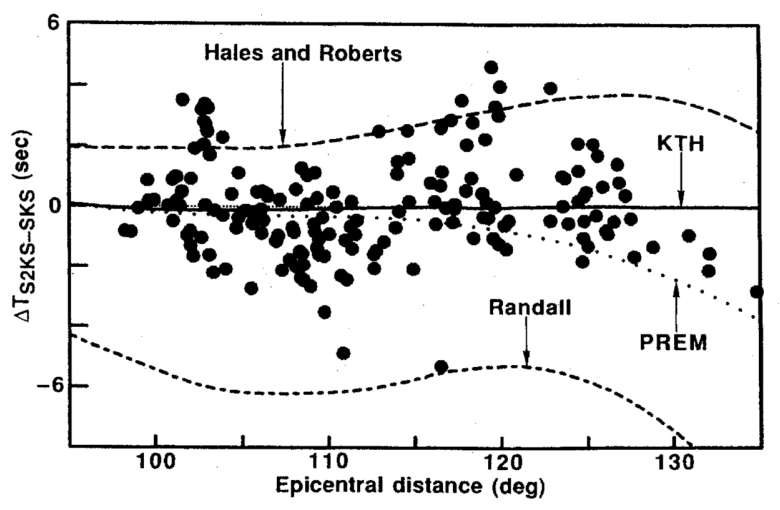

Fig. 7. Residuals of the differential travel times of S2KS-SKS as a function of epicentral distances, for the same models as in Fig. 6 with respect to Eq. (2a).

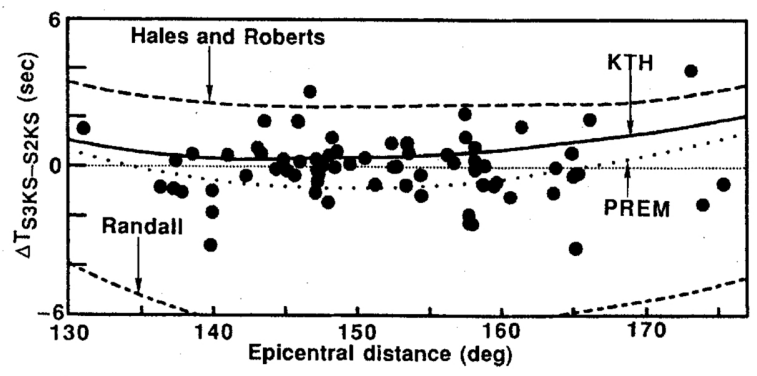

Fig. 8. Residuals of the differential travel times of S3KS-S2KS for the same models as in Fig. 6. Reference times is calculated from Eq. (2b).

$\Delta=110^{\circ}$ with increasing epicentral distances like as the observation of SoURIAU and PoUPINET (1991). Those from KTH do not show definite dependence for the epicentral distances. Figure 8 shows that the residuals $\Delta T_{S 3 K S-S 2 K S}$ of the RANDALL (1970) and HALES and ROBERTS (1971) with respect to Eq. (2b) are negative and positive, respectively. Those of KTH and PREM show small difference from each other.

It can be concluded that our model KTH explains fairly well not only the SKS travel times but also the differential travel times of S2KS-SKS and S3KS-S2KS compared with three other previous models.

\subsection{Effects of velocity structure in the lowermost mantle}

We derived above the velocity model in the outer core by subtracting the travel times in the mantle of the PREM model. Even now, the velocities in the lowermost mantle, i.e. the $\mathrm{D}^{\prime \prime}$ region, are somewhat controversial. The following models with different velocity gradient have been proposed for the $\mathrm{D}^{\prime \prime}$ region; negative velocity gradient (CLEARY, 1969; DooRnBos and MONDT, 1979; Doornbos, 1983), neutral (PREM), positive velocity gradient (MITCHELL and Helmberger, 1973; MUla, 1981) and the existence of a discontinuity (LAY and Helmberger, 1983; Young and Lay, 1987; Revenough and Jordan, 1991). Here, we discuss the effects of the structures of the $\mathrm{D}^{\prime \prime}$ region on the velocities in the outer core. Thus we use the following four 
S wave velocity models obtained by changing the velocity gradient in the lowermost $320 \mathrm{~km}$ of the mantle in the PREM: (1) model KTHF1: a strong positive gradient $\left(0.0014 \mathrm{sec}^{-1}\right)$, (2) KTHF2: a slightly positive gradient $\left(0.0007 \mathrm{sec}^{-1}\right)$, (3) KTHS1: a strong negative gradient $\left(-0.0009 \mathrm{sec}^{-1}\right)$, (4) KTHS2: a slightly negative gradient $\left(-0.0002 \mathrm{sec}^{-1}\right)$. A model with a discontinuity is not considered because we are interested in the trade-off between travel times in the core and mantle rather than a precise structure in the lowermost mantle.

Figure 9 shows the four $\mathrm{S}$ velocity models in the lowermost $320 \mathrm{~km}$ of the mantle and those of $\mathrm{P}$ waves in the outer core corresponding to the lowermost mantle models. The velocity perturbations in the lowermost mantle do affect on the $\mathrm{P}$ velocity structures in the outermost $200 \sim 300 \mathrm{~km}$ of the core. In general, a fast. $\mathrm{S}$ velocity in the lowermost mantle corresponds to a slow $\mathrm{P}$ velocity in the outermost core and vice versa. The model KTHF 1 shows $2 \%$ slower $\mathrm{P}$ velocity at the top of the core than KTH. KTHF2 shows $1 \%$ slower P velocity. KTHS1 shows $2 \%$ faster $\mathrm{P}$ velocity. KTHS2 shows a $1 \%$ faster $\mathrm{P}$ velocity. Therefore, if the $\mathrm{S}$ velocity at the base of the mantle has $\pm 5 \%$ ambiguity, the corresponding $\mathrm{P}$ velocity at the top of the core is affected by as much as $\pm 2 \%$ ambiguity.

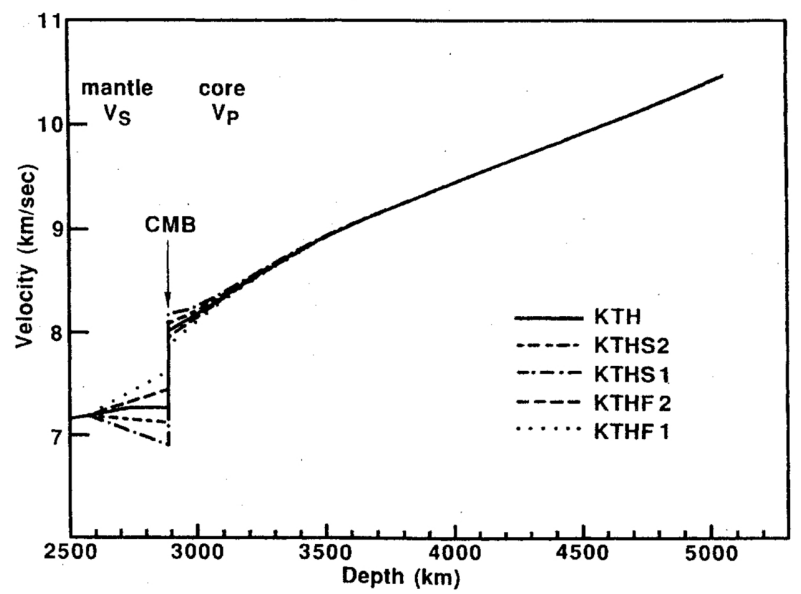

Fig. 9. Assumed $\mathrm{S}$ velocity structures in the lowermost $320 \mathrm{~km}$ of the mantle and the corresponding $\mathrm{P}$ velocities in the outer core. The velocity gradients in the lowermost mantle are strong positive (model KTHF1: $0.0014 \mathrm{sec}^{-1}$ ), slightly positive (KTHF2: $0.0007 \mathrm{sec}^{-1}$ ), strong negative (KTHS1: $-0.0009 \mathrm{sec}^{-1}$ ) and slightly negative (KTHS2: $-0.0002 \mathrm{sec}^{-1}$ ).

The strong positive velocity gradient in the $\mathrm{D}^{\prime \prime}$ region (MITCHELl and HELMBERGER, 1973) was based on the observation of amplitude ratios of ScS/S. This result is not the globally averaged one but is only based on local analysis for Central America. JORDAN and LYNN (1974) and LAY (1983) suggested that the middle of mantle beneath Central America is anomalous. LAY (1983) also suggested that the cause of the anomalies in the amplitude ratio of ScS/S is not due to the structure in the $\mathrm{D}^{\prime \prime}$ region. The strong negative velocity gradient in the $\mathrm{D}^{\prime \prime}$ region (CLEARY, 1969) was based on the observation of slowness of diffracted $P$ and $S$ waves. CLEARY's (1969) interpretation based on the ray theory was questioned because it is difficult to apply the ray theory to the diffracted waves. Analysis of amplitude decay and apparent velocity of the diffracted waves was done by MONDT (1977), Doornbos and MONDT (1979), using the full wave theory and by MUlA (1981), WYSESSION et al. (1992) using reflectivity method. These results support a slightly positive or slightly negative gradient as an average structure in the $\mathrm{D}^{\prime \prime}$ region. Thus, we can eliminate the extreme velocity models KTHS1 and KTHF1 with the strong positive and 
negative velocity gradients in the $\mathrm{D}^{\prime \prime}$ region.

\section{Discussion}

\subsection{Inhomogeneity index $\eta$}

In this section, we discuss physical conditions of the outer core based on the new velocity model KTH and those derived from different $\mathrm{D}^{\prime \prime}$ models. The most relevant parameter in the discussion of the physical conditions in the outer core is the inhomogeneity index $\eta$ expressed using seismic results as

$$
\eta=\frac{\mathrm{d} k}{\mathrm{~d} p}+g^{-1} \frac{\mathrm{d} \phi}{\mathrm{d} r}
$$

where $k$ is the incompressibility of the media, $p$ is the in-situ pressure, $g$ is the gravity acceleration, $r$ is the radial distance from the center of the Earth, and $\phi=v_{P}^{2}-4 v_{S}^{2} / 3$ (BULlen, 1975). The departure of the index $\eta$ from unity is considered to be a manifestation of non-adiabatic gradient of temperature or chemical inhomogeneity or a combination of both. These effects are shown as

$$
\eta=1+\frac{\phi}{g \rho} \frac{\partial \rho}{\partial Z} \frac{\mathrm{d} Z}{\mathrm{~d} p}-\frac{\alpha \phi \tau}{g}
$$

where $\rho$ is the medium density, $Z$ is a representative atomic number for a sufficient index of chemical composition, $\alpha$ is the thermal expansion coefficient, $\tau$ is a difference between the actual and adiabatic temperature gradient (BULLEN, 1975). BULLEN (1969) estimated $\eta$ in the outermost $700 \mathrm{~km}$ from the velocities by JeFFREYs (1939) and GUTENBERG (1951), indicating that the outermost $700 \mathrm{~km}$ of the core was in mild inhomogeneity $(\eta=1.4$ just beneath the CMB and gradually approaching to unity with increasing depth) and suggested the phase of material in the outermost $700 \mathrm{~km}$ was different from the rest of the outer core. However, this previous estimation should be revisited because the old Earth models did not have sufficient accuracy in the upper $1000 \mathrm{~km}$ of the core.

Figure 10 shows the index $\eta$ in the outermost $1000 \mathrm{~km}$ of the core as a function of radius for the three velocity models KTH, KTHF2, and KTHS2 using Eq. (4), where $\mathrm{d} k / \mathrm{d} p$ and $g$ being taken from the PREM. Within radius from 2500 to $2900 \mathrm{~km}$, the variations of $\eta$ calculated from each model are almost constant and constrained in the range between 1.1 and 1.2. We assume that physical condition in the middle of the outer core is adiabatic and homogeneous and the true value of $\eta$ is unity since convective motion is postulated by various dynamo theories. Therefore, we assume that $\eta$ in the radius $2500 \sim 2900 \mathrm{~km}$ calculated from the models KTH, KTHF2 and KTHS2 is a manifestation of adiabatic and homogeneous condition.

Here we describe two characteristics in the variation of $\eta$. First, average values of $\eta$ from the three versions of $\mathrm{KTH}$ model in the radius range of $2900 \sim 3300 \mathrm{~km}$ are about 0.2 lower than those in the $2500 \sim 2900 \mathrm{~km}$. The index $\eta$ in this region is possibly less than unity and a preferable value is 0.8 under the assumption that the region of radius $2500 \sim 2900 \mathrm{~km}$ is adiabatic and homogeneous. Second, $\eta$ begins to increase at the radius of $3200 \mathrm{~km}$ with increasing radius. In particular, $\eta$ of the KTH and KTHS2 in the outermost $100 \sim 200 \mathrm{~km}$ of the core exceeds the level of the adiabatic-homogeneous condition previously assumed. Since $2 \%$ inaccuracy of the structure of the $\mathrm{D}^{\prime \prime}$ still remains, we can conclude that the $\eta$ immediately beneath the CMB is slightly greater than unity and lies between 1.0 and 2.0 if the $\eta$ in the radius $2500 \sim 2900 \mathrm{~km}$ is assumed to be unity. A preferable value of the $\eta$ immediately beneath the CMB is 1.5 which is a mean value of our result.

The index $\eta$ was related to the Brunt-Väisälä frequency $N$ by

$$
N^{2}=\frac{g^{2}}{\phi}(\eta-1)
$$




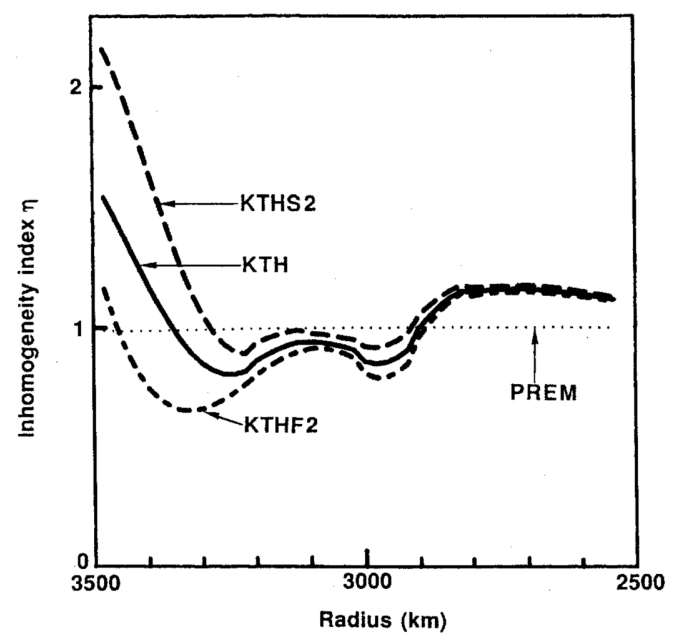

Fig. 10. Variations of the index $\eta$ in the outermost $1000 \mathrm{~km}$ of the core for the KTH (solid line), KTHS2 (long dashed line) and KTHF2 (dashed line) models.

(e.g. MASTERS, 1979). When we ignore viscid and pressure terms in the equation of motion, $N^{2}$ satisfies the equation $\partial^{2} \omega / \partial t^{2}+N^{2} \omega=0$ (e.g. GILL, 1982), where $\omega$ is the vertical displacement. The index $\eta$ less than unity in the radius $2900 \sim 3300 \mathrm{~km}$ yields to negative $N^{2}$. Therefore, $N$ is imaginary and can be interpreted as an exponential growth rate of instability for a fluid in the outer core, suggesting a vigorous convection. The outermost $100 \sim 200 \mathrm{~km}$ of the core is possibly in the condition of $\eta>1$. If preferable value of $\eta=1.5$ is correct, $N$ is real and angular frequency of $8.6 \times 10^{-4} \mathrm{~s}^{-1}$ corresponding to the period $2 \pi / N$ of 2.0 hours. The many values of $N$ in the outer core has been proposed based on the free oscillation and the Earth tide and they vary from $0.9 \times 10^{-4}$ to $6.0 \times 10^{-4} \mathrm{~s}^{-1}$ (see MELCHIOR, 1986). Recently, BRAGINSKY (1992) proposes $N=2 \Omega$, where $\Omega$ is the frequency of the Earth's rotation $\left(\Omega=7.3 \times 10^{-5} \mathrm{~s}^{-1}\right)$. The $N$ of the present study is slightly larger than the maximum estimate of the previous studies. The ratio of $N^{2}$ to $4 \Omega^{2}\left(A=N^{2} / 4 \Omega^{2}\right)$ is used to consider the degree of the stratification in the core (OLSON, 1977). Stratification in the core is strongly stable when $A>1$, and is weakly stable when $A<1$. The previous estimates of $N$ are $0.9 \times 10^{-4}$ to $6.0 \times 10^{-4} \mathrm{~s}^{-1}$ and our estimate is $8.6 \times 10^{-4} \mathrm{~s}^{-1}$, they correspond to the values of $A$ being 0.38 to 16.9 (MelCHIOR, 1986) and 35, respectively. Therefore, we suggest that stratification in the outermost $100 \sim 200 \mathrm{~km}$ of the core is strongly stable.

Possible mechanisms for the formation of stratification are chemical inhomogeneity, nonadiabatic gradient of temperature and the combination of both (BULLEN, 1975). If the region is adiabatic but chemically inhomogeneous, the total density increases in the outermost $200 \mathrm{~km}$ of the core is $5 \%$ while that in the PREM is $3 \%$. If the region is homogeneous but non-adiabatic, the non-adiabatic temperature gradient $\tau$ is about $-8 \mathrm{~K} / \mathrm{km}$ when $\alpha=10^{-5}$.

Some mechanisms for the formation of the stably stratified layer has been discussed in reference to the estimation of thickness of the layer. Assuming light material released from the inner-core boundary (ICB) might be stored at the top of the core, FEARN and LOPER (1981) proposed the layer having the thickness of $70 \mathrm{~km}$. BRAGINSKY (1984) gave that thickness of the layer of light fluid, which he called the H-layer, was $20 \mathrm{~km}$ based on the geomagnetic consideration. Recently, BRAGINSKY (1992) revised thickness of the H-layer to be $80 \mathrm{~km}$ assuming that eigen-oscillations of the H-layer was similar to Magnetic-Archmedean-Coriolis (MAC) waves. It 
is interesting to point out that the above theoretical estimates of the thickness are not far from the seismological estimates of about $100 \mathrm{~km}$ thickness shown in the present study and in LAY and YounG's (1990) model. Based on the assumption that the thermal convection in the core was caused by the global cooling of the Earth, GuBBINs et al. (1982) showed the outermost 100 1000 $\mathrm{km}$ of the core was possibly stratified.

\subsection{Temperature distribution just beneath the $C M B$}

It is difficult to distinguish whether stably stratified layer in the outermost core results from chemical inhomogeneity or non-adiabatic gradient of temperature using only the radial structure of the core. Thus, other information such as lateral structure should be taken into account. Previously homogeneity of the outer core has been supported by observation of PnKP phases which pass the whole outer core multiply reflecting under the CMB for $(n-1)$ times (BUCHIBINDER, 1972), and by that of PKP phases of which deepest points are lower part of the outer core (MORELli and DzIEWONSKI, 1987). However, possibility of heterogeneity just beneath the CMB is not completely removed (e.g. SteVEnson, 1987). Recently, based on seismological studies, lateral heterogeneity in the outermost core is discussed and suggested that $\mathrm{P}$ velocity anomaly in the outermost core is $\pm 0.3 \%$ (Souriau and Poupinet, 1990; TANaka and Hamaguchi, 1993a). And studies of the geomagnetic secular variations reveal fluid flow and temperature distribution at the top of the core (for a review, BLOXHAM and JACKSON, 1991). Here we focus our attention to a pattern of degree 1 which is found in the results of both seismological (TANAKA and HAMAGUCHI, 1993a) and geomagnetic (BloxhAM and JACKSON, 1991) studies.

TANAKA and HAMAGUCHI (1993a), who analyzed geographical distribution of S2KS-SKS and S3KS-S2KS travel time residuals using spherical harmonics, found that a pattern of degree 1 was dominant in the residuals which could not be explained by any of the known CMB structures (Fig. 11). Average residuals are $+0.6 \mathrm{~s}$ in the Pacific (P-) hemisphere and $-0.6 \mathrm{~s}$ in the Atlantic-Africa-India (A-) hemisphere. They interpreted that this hemispherical pattern resulted
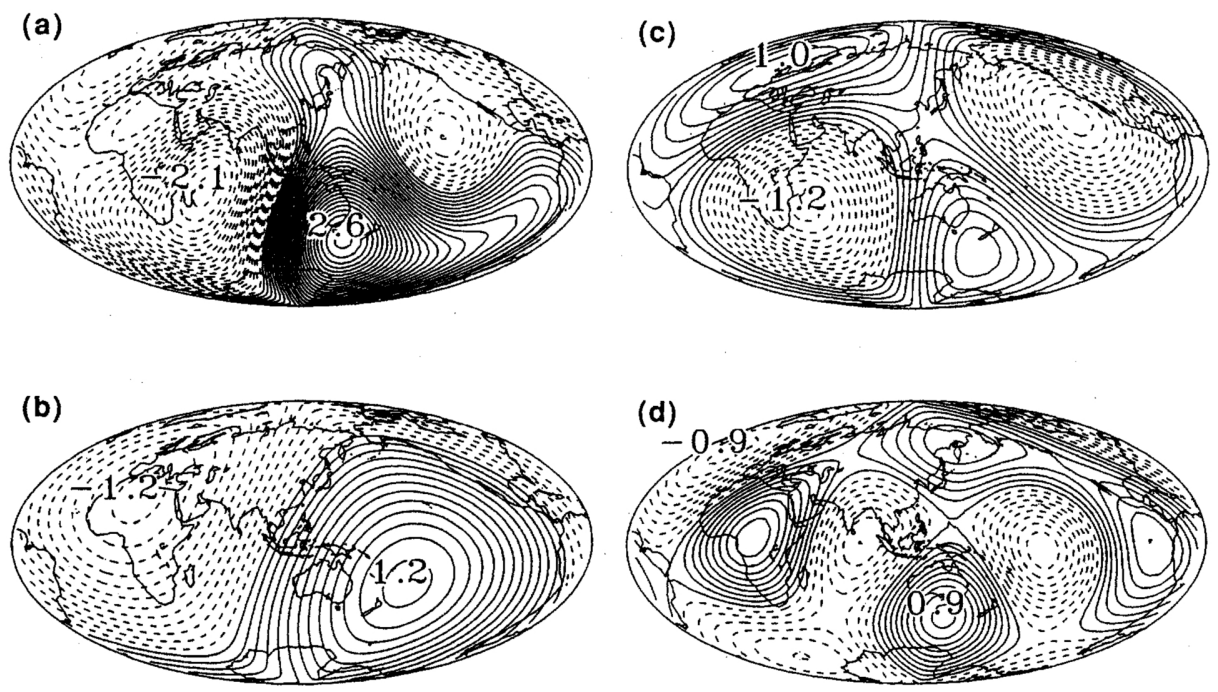

Fig. 11. Geographical distributions of residuals of $\Delta T_{S 2 K S-S K S}$ and $\Delta T_{S 3 K S-S 2 K S}$ represented by spherical harmonics (a) up to degree 3 , (b) degree 1, (c) degree 2, and (d) degree 3 components. Solid and broken lines mean positive and negative values, respectively. Contour interval is $0.1 \mathrm{sec}$. Map is in Aitof projection centered on $\left(0^{\circ}, 120^{\circ} \mathrm{E}\right)$ (after TANAKA and HAMAGUCHI, 1993a). 
from $\mathrm{P}$ velocity anomaly $-0.3 \%$ in the $\mathrm{P}$-hemisphere and $+0.3 \%$ in the A-hemisphere in the outermost $200 \mathrm{~km}$ of the core. The temperature distribution at the top of the core inferred from the geomagnetic study showed that a degree 1 component is dominant and that temperature is high in the A-hemisphere and low in the P-hemisphere (Figs. 9 and 10 of BLOXHAM and JACKSON, 1991). Patterns of the degree 1 found in the $P$ wave velocity heterogeneity and in the temperature distribution are quite similar to each other. TANAKA and HAMAGUCHI (1993a) described the translation from the velocity heterogeneity into the temperature distribution. Here we recalculate the temperature distribution using the velocity model determined in the present study, and consider two extreme cases for interpretation of the $\eta$ departure from unity.

If the outermost core in the $\mathrm{P}$ - and A-hemispheres is assumed to be chemically inhomogeneous, temperature difference between two hemispheres results from difference of adiabatic gradients of temperature in the two hemispheres. Adiabatic gradient of temperature in terms of seismic velocity is given by

$$
\left(\frac{\partial T}{\partial r}\right)_{S}=-\frac{g \gamma T}{\phi}
$$

(Melchior, 1986) where $T$ is the adiabatic temperature, $S$ is the entropy, and $\gamma$ is the Grüneisen parameter. The integration of Eq. (7) yields

$$
\ln \frac{T_{C M B}}{T_{r e f}}=-\int_{r_{r e f}}^{r_{C M B}} \frac{g \gamma}{\phi} \mathrm{d} r
$$

where $T_{C M B}$ is the adiabatic temperature at the CMB, $T_{r e f}$ is that at a reference radius of $r_{r e f}$, $r_{C M B}$ is the radius of the CMB. Assuming $\gamma \approx 1.5$ through the outer core and using $v_{P}$ of the $\mathrm{KTH}$ model and considering $\pm 0.3 \%$ perturbations of $\mathrm{P}$ velocity for the $\mathrm{P}$ - and A-hemispheres, $T_{C M B} / T_{r e f}$ is calculated for each hemisphere. When $r_{C M B}-r_{r e f}=200 \mathrm{~km}$, for a maximum range discussed in the previous section, is assumed,

$$
\begin{aligned}
& T_{C M B} / T_{r e f}=0.95373 \quad \text { for the P-hemisphere, } \\
& T_{C M B} / T_{r e f}=0.95425 \text { for the A-hemisphere. }
\end{aligned}
$$

Therefore, $T_{C M B}$ in the A-hemisphere is higher than that in the P-hemisphere if $T_{r e f}$ is laterally uniform through the core. The temperature differences between the two hemispheres at the CMB are

$$
\Delta T_{C M B}=T_{C M B}(A)-T_{C M B}(P)=5.2 \times 10^{-4} T_{r e f} .
$$

If $T_{r e f}$ is assumed to be $3000 \mathrm{~K}, \Delta T_{C M B}=1.6 \mathrm{~K}$.

If the outermost core is assumed to be homogeneous, temperature difference between the two hemispheres results from those of non-adiabatic temperature gradients. Temperature is estimated from the integration of $(\mathrm{d} T / \mathrm{d} r)$ given by

$$
\begin{aligned}
T_{C M B} & =\int_{r_{r e f}}^{r_{C M B}} \frac{\mathrm{d} T}{\mathrm{~d} r} \mathrm{~d} r \\
& =-\int_{r_{r e f}}^{r_{C M B}} \tau \mathrm{d} r+\int_{r_{r e f}}^{r_{C M B}}\left(\frac{\partial T}{\partial r}\right)_{S} \mathrm{~d} r \\
& =-\int_{r_{r e f}}^{r_{C M B}} \frac{g}{\alpha \phi}(1-\eta) \mathrm{d} r+\int_{r_{r e f}}^{r_{C M B}}\left(\frac{\partial T}{\partial r}\right)_{S} \mathrm{~d} r .
\end{aligned}
$$

Since $\eta$ and $\alpha$ are quantities with uncertainty, only rough evaluation is considered. If $\eta=1.5$, and $\alpha=10^{-5}$, and $g$ and $\phi$ are assumed to be constants with the value at the top of the core, $T_{C M B}$ is given by

$$
T_{C M B}=-\frac{g}{\alpha \phi}(1-\eta) \times\left(r_{C M B}-r_{r e f}\right)+T_{\text {adiabat }}+T_{r e f}
$$


where $T_{\text {adiabat }}$ is a temperature increase from adiabatic gradient of temperature. $T_{C M B}$ is calculated for each hemisphere involving $\pm 0.3 \% \mathrm{P}$ wave heterogeneities as follows:

$$
\begin{array}{ll}
T_{C M B}=1673 \mathrm{~K}+T_{\text {adiabat }}+T_{\text {ref }} & \text { for the P-hemisphere, } \\
T_{C M B}=1653 \mathrm{~K}+T_{\text {adiabat }}+T_{\text {ref }} & \text { for the A-hemisphere, }
\end{array}
$$

when $r_{C M B}-r_{r e f}=200 \mathrm{~km}$. Therefore, $T_{C M B}$ in the A-hemisphere is lower than that in the P-hemisphere if $T_{\text {adiabat }}$ and $T_{\text {ref }}$ are laterally uniform through the core. The temperature difference between the two hemispheres at the CMB is

$$
\Delta T_{C M B}=T_{C M B}(A)-T_{C M B}(P)=-20 \mathrm{~K} \text {. }
$$

We found that the sign of temperature distribution is dependent on the physical condition in the core. If the outermost core is chemically inhomogeneous but adiabatic, $T_{C M B}$ in the A-hemisphere is higher than that in the P-hemisphere. If the outermost core is homogeneous but non-adiabatic, the pattern of $T_{C M B}$ distribution shows an opposite sign compared with the former one. We could not determine a preferable pattern of temperature distribution using seismic information only. If the temperature distribution from the geomagnetic study (BLOXHAM and JACKSON, 1991) is correct, chemically inhomogeneous and adiabatic conditions are preferred in the outermost core. This circumstance readily leads to a chemically stratified layer existing in the outermost $100 \sim 200 \mathrm{~km}$ of the core. However, it is not clear yet whether the stratified layer originates from accumulation of iron released at the ICB (FEARN and LOPER, 1981), or that of material reaction with the lowermost mantle (KNITTLE and JEANLOZ, 1989) or other unknown mechanisms. Therefore further analyses of the base of the mantle and the ICB is needed for understanding of the origin of the stably stratified layer in the outermost core.

\section{Conclusions}

We derived a seismic velocity model named KTH in the outer core using 229 travel times of SKS and 174 travel times of S2KS phases recorded by widely distributed seismic stations. Travel time corrections for mantle heterogeneities and ellipticity were applied. We found that the velocity at the top of the core is $8.016 \mathrm{~km} \mathrm{~s}^{-1}$, and the velocity in the outermost $200 \mathrm{~km}$ of the core is about $0.11 \mathrm{~km} \mathrm{~s}^{-1}$ faster than those of HALES and ROBERTS (1971), while the velocity in the outer $200 \sim 400 \mathrm{~km}$ of the core is identical within $1 \%$ to those of Hales and Roberts. We examined effects of the structural uncertainty of the lowermost mantle on the velocity model in the core and found that $P$ velocity at the top of the core may be subject to $\pm 2 \%$ changes when the $\mathrm{S}$ velocity at the base of the mantle has $\pm 5 \%$ ambiguity.

Based on the new velocity model KTH, we discussed stable stratification in the outermost core. The results are summarized as follows:

1. Inhomogeneity index $\eta$ related to Brunt-Väisälä frequency $N$ is slightly greater than unity within $100 \sim 200 \mathrm{~km}$ in the outermost core, if we exclude the extreme case where the $\mathrm{S}$ wave velocity at the base of the mantle is faster than that of the PREM model by more than $2 \%$. A preferable value of $\eta$ just beneath the CMB is 1.5 corresponding to $N$ of $8.6 \times 10^{-4} \mathrm{~s}^{-1}$. This suggests that the outermost $100 \sim 200 \mathrm{~km}$ of the core is stably stratified.

2. The above thickness of stably stratified layer is not far from those of $70 \sim 80 \mathrm{~km}$ thickness estimated from theoretical considerations.

3. The estimate of temperature distribution at the top of the core suggests that a chemically stratified layer in the outermost core is preferred on the basis of the geomagnetic study.

We thank two anonymous reviewers for valuable comments. This work is partly supported by Grantin-Aid for Scientific Research (B) No. 03452055 and partly by a grant under the Monbusho International Scientific Research Program No. 04041018. 


\section{REFERENCES}

BloxhAM, J. and D. GubBins, Thermal core-mantle interactions, Nature, 325, 511-513, 1985.

Bloxham, J. and A. JACKSON, Fluid flow near the surface of Earth's outer core, Rev. Geophys., 29, 97-120, 1991.

BraginSKY, S. I., Short-periodgeomagnetic secular variation, Geophys. Astrophys. Fluid Dyn., 30, 1-78, 1984.

Braginsky, S. I., Events on the core-mantle boundary, and the geodynamo, The Third SEDI Symposium, Mizusawa, Japan, July 6-10, 1992.

Buchibinder, G. G. R., Travel times and velocities in the outer core from PmKP, Earth Planet. Sci. Lett., 14, $161-168,1972$.

Bullen, K. E., Compressibility-pressure gradient and constitution of the Earth's outer core, Geophys. J. R. astr. Soc., 18, 73-79, 1969.

Bullen, K. E., The Earth's Density, 420 pp., Chapman and Hall, London, 1975.

Bullen, K. E. and R. A. W. HADDON, The ellipticities of surfaces of equal density inside the Earth, Phys. Earth Planet. Inter., 7, 199-202, 1973.

ChоY, G. L., Theoretical seismograms of core phases calculated by frequency-dependent full wave theory, and their interpretation, Geophys. J. R. astr. Soc., 51, 275-312, 1977.

ChOY, G. L. and P. G. Richards, Pulse distortion and Hilbert transformation in multiply reflected and refracted body waves, Bull. Seismol. Soc. Am., 65, 55-70, 1975.

Cleary, J., The S velocity at the core-mantle boundary, from observations of diffracted S, Bull. Seismol. Soc. Am., 59, 1399-1405, 1969.

Doornbos, D. J., Present seismic evidence for a boundary layer at the base of the mantle, J. Geophys. Res., 88, 3498-3505, 1983.

Doornios, D. J. and J. C. MondT, P and S waves diffracted around the core and the velocity structure at the base of the mantle, Geophys. J. R. astr. Soc., 57, 381-395, 1979.

Dziewonski, A. M., Mapping the lower mantle: Determination of lateral heterogeneity in P velocity up to degree and order 6, J. Geophys. Res., 89, 5929-5952, 1984.

Dziewonski, A. M. and D. L. Anderson, Preliminary reference Earth model, Phys. Earth Planet. Inter., 25, 297-356, 1981.

DziewONSKI, A. M. and F. GILBERT, The effect of small, aspherical perturbations on travel times and a reexamination of the corrections for ellipticity, Geophys. J. R. astr. Soc., 44, 7-17, 1976.

FEARN, D. R. and D. E. Loper, Compositional convection and stratification of the Earth's core, Nature, 289, 393-394, 1981.

Gill, A. E., Atomosphere-Ocean Dynamics, 662 pp., Academic Press, New York, 1982.

Gubbins, D., C. J. Thomson, and K. A. Whaler, Stable regions in the Earth's liquid core, Geophys. J. R. astr. Soc., 68, 241-251, 1982.

Gutenberg, B., PKKP, P'P', and the Earth's core, Trans. Am. Geophys. Un., 32, 373-390, 1951.

Hales, A. L. and J. L. RoBerts, The velocities in the outer core, Bull. Seismol. Soc. Am., 61, 1051-1059, 1971.

JAULT, D. and J.-L. LE MOUËL, Core-mantle boundary shape: constraints inferred from the pressure torque acting between the core and mantle, Geophys. J. Int., 101, 233-241, 1990.

JEANLOZ, R., The nature of the Earth's core, Ann. Rev. Earth Planet. Sci., 18, 357-386, 1990.

Jeffreys, H., The times of the core waves, Mon. Not. R. astr. Soc., Geophys. Suppl., 4, 548-561, 1939.

Jordan, T. H. and W. S. LYNN, A velocity anomaly in the lower mantle, J. Geophys. Res., 79, 2679-2685, 1974.

Kohler, M. D. and T. TAnimoto, One-layer global inversion for outermost core velocity, Phys. Earth Planet. Inter., 72, 173-184, 1992.

KIND, R. and G. MüLLER, The structure of the outer core form SKS amplitudes and travel times, Bull. Seismol. Soc. Am., 67, 1541-1554, 1977.

KNittle, E. and R. Jeanloz, Simulating the core-mantle boundary: an experimental study of high-pressure reactions between silicates and liquid iron, Geophys. Res. Lett., 16, 609-612, 1989.

LAY, T., Localized velocity anomalies in the lower mantle, Geophys. J. R. astr. Soc., 72, 483-516, 1983.

LAY, T., Structure of the core-mantle transition zone: A chemical and thermal boundary layer, EOS, Trans. Am. Geophys. Un., 70, 49-59, 1989.

LAY, T. and D. V. Helmberger, A lower mantle S-wave triplication and the shear velocity structure of $\mathrm{D}^{\prime \prime}$, Geophys. J. R. astr. Soc., 75, 799-838, 1983.

LAY, T. and C. J. Young, The stable-stratified outer most core revisited, Geophys. Res. Lett., 17, 2001-2004, 1990.

LOPER, D. E., Mantle plumes, Tectonophysics, 187, 373-384, 1991.

MASTERS, G., Observation constraints on the chemical and thermal structure of the Earth's deep interior, Geophys. J. R. astr. Soc., 57, 507-534, 1979.

Melchior, P., The Physics of the Earth's Core: An Introduction, 256 pp., Pergamon Press, Oxford, 1986.

Mitchell, B. J. and D. V. Helmberger, Shear velocities at the base of the mantle from observations of S and $\mathrm{ScS}$, J. Geophys. Res., 78, 6009-6020, 1973.

Mondt, J. C., SH waves: Theory and observations for epicentral distances greater than 90 degrees, Phys. Earth Planet. Inter., 15, 46-59, 1977. 
Morelli, A. and A. M. DzIEwonski, Topography of the core-mantle boundary and lateral homogeneity of the liquid core, Nature, 325, 678-683, 1987.

MulA, A. H. G., Amplitudes of diffracted long-period P and S waves and the velocities and $Q$ structure at the base of the mantle, J. Geophys. Res., 86, 4999-5011, 1981.

Olson, P., Internal waves in the earth's core, Geophys. J. R. astr. Soc., 51, 183-215, 1977.

RANDALL, M. J., SKS and seismic velocities in the outer core, Geophys. J. R. astr. Soc., 21, 441-445, 1970.

Revenough, J. and T. H. Jordan, A study of mantle layering beneath the Western Pacific, J. Geophys. Res., 94, $5787-5813,1989$.

Revenough, J. and T. H. JoRdAn, Mantle layering from ScS reverberations 4. The lower mantle and core-mantle boundary, J. Geophys. Res., 96, 19811-19824, 1991.

SASAO, T. and J. M. WAHR, An excitation mechanism for the free 'core nutation', Geophys. J. R. astr. Soc., 64, $729-746,1981$.

Souriau, A. and G. Poupinet, A latitudinal pattern in the structure of the outermost liquid core, revealed by the travel times of SKKS-SKS seismic phases, Geophys. Res. Lett., 17, 2005-2007, 1990.

Souriau, A. and G. PoupINET, A study of the outermost liquid core using differential travel times of SKS, SKKS and S3KS phases, Phys. Earth Planet. Inter., 68, 183-199, 1991.

StEvenson, D. J., Limits on lateral density and velocity variations in the Earth's outer core, Geophys. J. R. astr. Soc., 88, 311-319. 1987.

Su, W. and A. M. DzIEwonski, Predominance of long-wavelength heterogeneity in the mantle, Nature, 352, 121-126, 1991.

TANAKA, S. and H. HAMAGUCHI, Degree one heterogeneity at the top of the Earth's core, revealed by SmKS travel times, in Dynamics of Earth's Deep Interoir and Earth Rotation, IUGG vol. 12, AGU Geophys. Monogr. vol. 72, edited by J.-L. Le Mouël, D. Smylie, and T. Herring, pp. 127-134, Amer. Geophys. Union, Washington, D. C., 1993a.

TANAKA, S. and H. HAMAguCHI, Travel times and velocities in the outer core based on the global observation of SmKS seismic phases, Tohoku Geophys. J., 34, 55-87, 1993b.

Wysession, M. E., E. A. OKAL, and C. R. BinA, The structure of the core-mantle boundary from diffracted waves, J. Geophys. Res., 97, 8749-8764, 1992.

Young, C. J. and T. LAY, Evidence for a shear velocity discontinuity in the lower mantle beneath India and the Indian Ocean, Phys. Earth Planet. Inter., 49, 37-53, 1987. 